\title{
Two-dimensional effects in laser-created plasmas measured with soft-x-ray laser interferometry
}

\author{
J. Filevich, J. J. Rocca, E. Jankowska, ${ }^{*}$ E. C. Hammarsten, K. Kanizay, and M. C. Marconi ${ }^{\dagger}$ \\ Department of Electrical and Computer Engineering, Colorado State University, Fort Collins, Colorado 80523 \\ S. J. Moon \\ Lawrence Livermore National Laboratory, Livermore, California 94551 \\ V. N. Shlyaptsev \\ Department of Applied Science, University of California Davis-Livermore, Livermore, California 94551
}

(Received 13 June 2002; published 29 May 2003)

\begin{abstract}
Soft-x-ray laser interferograms of laser-created plasmas generated at moderate irradiation intensities (1 $\times 10^{11}-7 \times 10^{12} \mathrm{~W} \mathrm{~cm}^{-2}$ ) with $\lambda=1.06 \mu \mathrm{m}$ light pulses of $\sim 13$-ns-FWHM (full width at half maximum) duration and narrow focus $(\sim 30 \mu \mathrm{m})$ reveal the unexpected formation of an inverted density profile with a density minimum on axis and distinct plasma sidelobes. Model simulations show that this strong twodimensional hydrodynamic behavior is essentially a universal phenomena that is the result of plasma radiation induced mass ablation and cooling in the areas surrounding the focal spot.
\end{abstract}

DOI: 10.1103/PhysRevE.67.056409

PACS number(s): 52.50.Jm, 42.55.Vc, 52.65.Kj, 52.70.La

The understanding of the dynamics of laser-created plasmas is of fundamental and practical interest. The hydrodynamic motion of plasmas created by laser irradiation of solid targets is conventionally known to result in electron density distributions with maximum density along the axis of the irradiation beam. However, in plasmas generated with high irradiation intensities the ponderomotive force has been observed to cause a density depression or cavity in the electron density profile. Early interferometry experiments of lasercreated plasmas at irradiation intensities of $3 \times 10^{14}$ $\mathrm{W} \mathrm{cm}^{-2}$ showed a flattening of the interfering fringes in the subcritical region that, for an axis-symmetric plasma, is indicative of a density depression [1]. Density depressions induced by the ponderomotive force have also been observed in numerous other high-intensity laser experiments, in agreement with simulations. Some of the most recent studies include the formation of plasma channels and laser-hole boring in underdense and overdense plasmas motivated by the fast ignitor concept in inertial confinement fusion [2,3]. These experiments involved laser intensities of $1.7 \times 10^{15}$ and 2 $\times 10^{17} \mathrm{~W} \mathrm{~cm}^{-2}$, respectively. In addition, for several cases involving short laser pulses the saturation of the heat flux, refraction, or channeling of the laser radiation due to relativistic self-focusing at ultrahigh fluxes are found to be responsible for density suppression [4,5].

In this paper, we report the observation of a pronounced density minimum on axis in both line-focus and spot-focus plasmas, generated at intensities as low as $10^{11} \mathrm{~W} \mathrm{~cm}^{-2}$, which cannot be explained by the ponderomotive force, the effects of laser radiation refraction, electron heat saturation, nor the influence of plasma instabilities. Our case is different, yet universal enough to exist in a wide parameter space.

\footnotetext{
*Permanent address: Dept. of Physics, Wroclaw University of Technology, Poland.

${ }^{\dagger}$ Permanent address: Dept. of Physics, University of Buenos Aires, Argentina.
}

In fact, in retrospective, evidence of these effects may be inferred from published visible laser plasma interferograms mapping much smaller electron densities of the order of $\sim 10^{18} \mathrm{~cm}^{-3}[6,7]$. However, this kind of two-dimensional (2D) plasma behavior was neither clearly revealed from the data nor understood. In our measurements, the $2 \mathrm{D}$ effect is distinctively uncovered by the use of soft-X-ray laser (SXRL) interferometry, which for the case of the spot-focus plasma experiment allowed us to map the density profile up to $\sim 10^{21} \mathrm{~cm}^{-3}$, close to the critical density. Simulations show that plasma radiation and heat conduction enlarge the ablation region creating a plasma density profile with large distinctive sidelobes and a minimum on axis.

SXRL interferometry is a powerful new tool for the diagnostics of dense plasmas, which allows probing of plasma densities and scale lengths larger than those that can be probed with optical lasers. Interferometry measurements of dense large scale plasmas conducted using a laboratory SXRL pumped by the Nova laser [8,9] significantly exceeded the density values probed with optical lasers, but were lim-

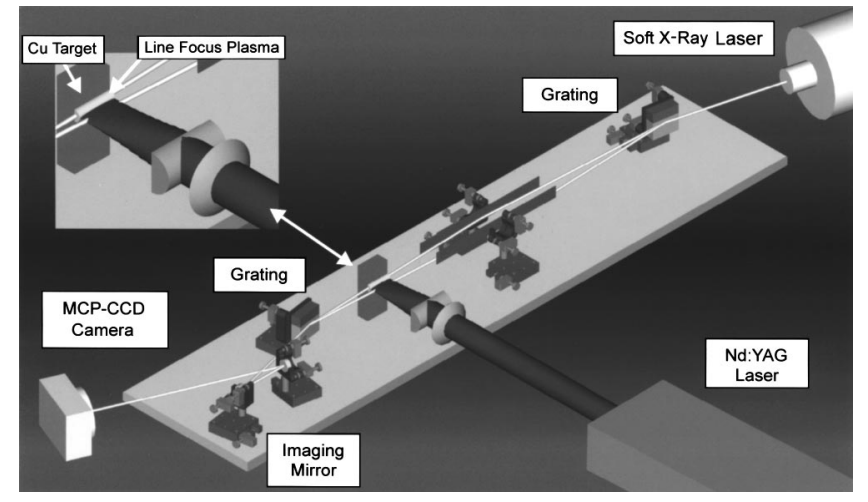

FIG. 1. Schematic representation of the experimental setup. The probe beam propagates along the axis of the line focus. The interferometer was positioned $\sim 2 \mathrm{~m}$ from the exit of the SXRL. The detector was placed $\sim 7 \mathrm{~m}$ from the interferometer. 


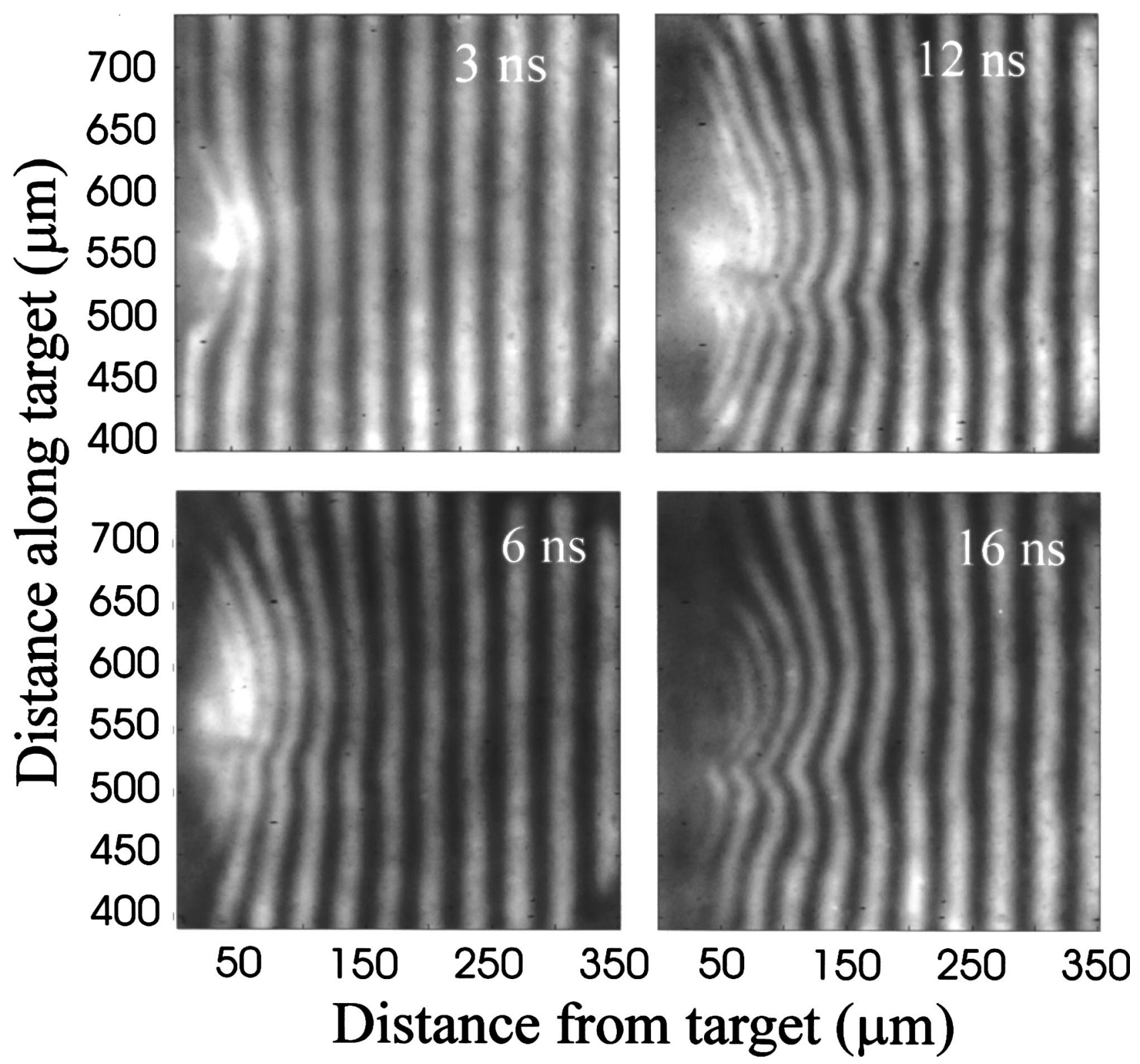

FIG. 2. Sequence of soft-x-ray interferograms describing the evolution of 1.8-mm-long plasma generated by focusing a 13-ns-FWHM $\mathrm{Nd}$ :YAG laser pulse with $0.6 \mathrm{~J}$ energy onto a $\sim 30-\mu \mathrm{m}$-wide line on a copper target. The time delays are measured with respect to the beginning of the laser pulse. The heating laser is incident from the right.

ited to several shots per day by the low repetition frequency of the pump laser. The results reported herein were obtained with a new soft-X-ray interferometry tool based on a portable SXRL and a Mach-Zehnder interferometer configuration in which diffraction gratings are used as beam splitters [10]. The high repetition rate of the probe laser allowed us to complete several series of interferograms of laser-created plasmas that map the entire temporal plasma evolution.

The experimental setup used in the measurements is shown in Fig. 1. It comprises a $\lambda=1.06 \mu \mathrm{m} Q$-switched Nd:YAG (yttrium aluminum garnet) laser (Quanta Ray GCR190) used to irradiate the target, the SXRL probe, and a soft-x-ray interferometer. The Ne-like Ar 46.9-nm capillary discharge laser, which has excellent spatial coherence [11], was described in previous publications $[12,13]$. SXRL pulses with a full width at half maximum (FWHM) duration of $\sim 1.2 \mathrm{~ns}$, energies of $\sim 0.1 \mathrm{~mJ}$, and beam divergence of $\sim 4.5$ mrad were used in the experiment. The interferometer uses gold-coated diffraction gratings as beam splitters [10], positioned at an incidence angle of $79^{\circ}$. It has the advantages of high throughput ( $\sim 6 \%$ per arm excluding imaging mirrors) and increased robustness against plasma debris. Elongated gold-coated grazing incidence mirrors are used to direct the beams towards the second grating, where they are recombined. Spherical mirrors coated with Si-Sc multilayers (normal incidence reflectivity of $\sim 40 \%$ ) [14] were used to image the plasma onto a charge coupled device with a microchannel plate $(\mathrm{MCP})$ detector with a magnification of either $51.2 \mathrm{x}$ or 24.7x. The line focus plasmas were generated by focusing the beam of the Nd:YAG laser (using a 30-cm- 


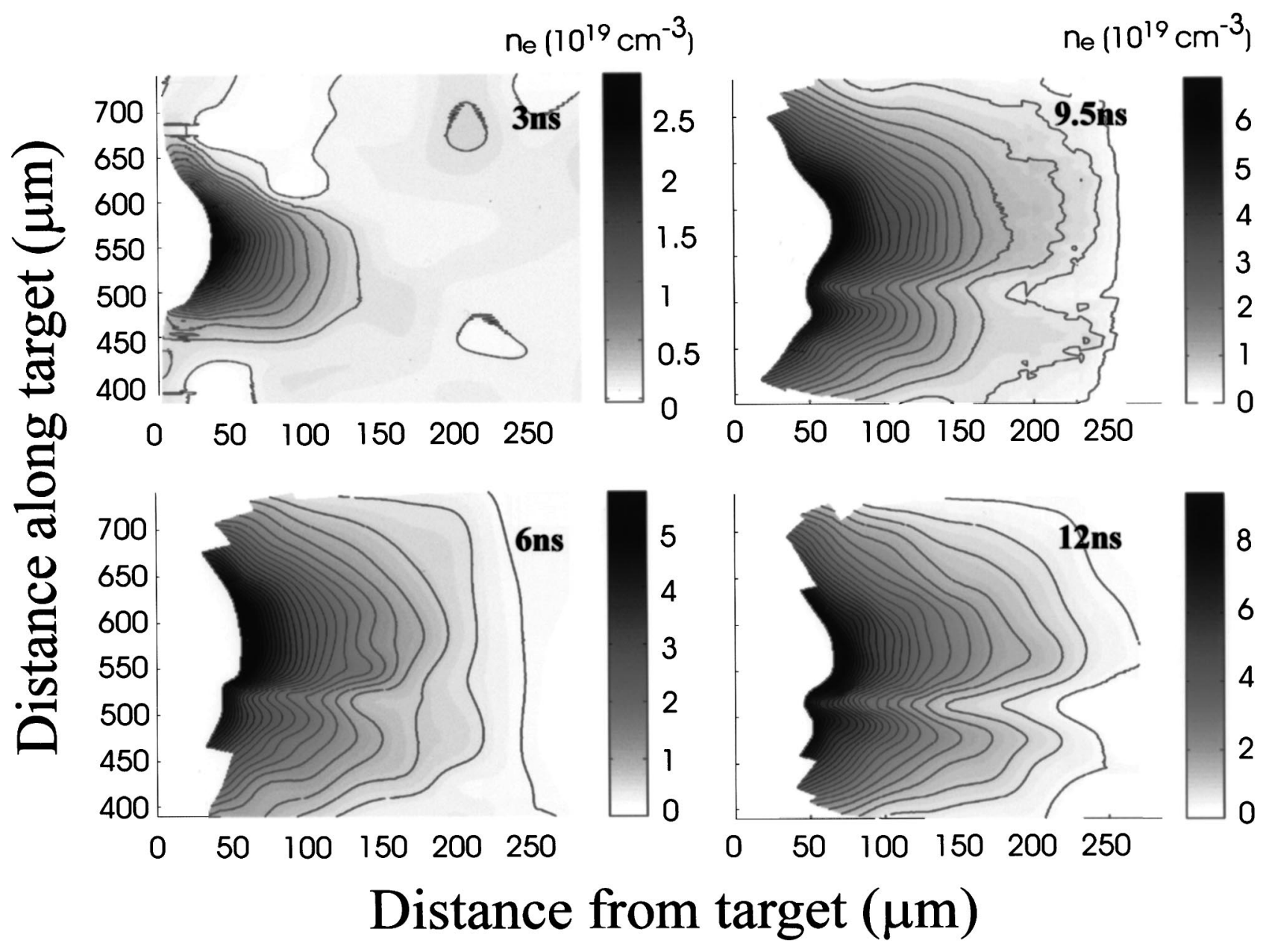

FIG. 3. Electron density profiles obtained from the interferograms.

focal-length cylindrical lens and a 10-cm-focal-length spherical lens) onto a polished electrolytic copper target placed in the zero-order arm of the interferometer. A system of pinholes was used to reduce the strong plasma selfemission collected by the detector. The detector's MCP was gated to further reduce the background radiation produced by the plasma self-emission. The firing of the SXRL was synchronized with respect to the Nd:YAG laser using a common master clock and appropriate delays. However, since the jitter in the firing of the SXRL was significant nearly 100 shots per series were required to map the entire plasma evolution with $\sim 1 \mathrm{~ns}$ resolution. The fringe visibility of the interferograms obtained without the plasma present exceeds 50\% across the entire $400 \times 500 \mu \mathrm{m}^{2}$ field of view.

Figure 2 shows a series of interferograms that depict the evolution of a line focus plasma created at an irradiation intensity of $0.9 \times 10^{11} \mathrm{~W} \mathrm{~cm}^{-2}$ by focusing $0.63 \mathrm{~J}-13$-nsFWHM duration laser pulses into a $\sim 30-\mu \mathrm{m}$-wide line 1.8 $\mathrm{mm}$ in length. These interferograms were obtained with a magnification of $24.7 \times$. Figure 3 shows the corresponding density maps obtained from the fringe shifts assuming the plasma is uniform along the probe beam axis. The earlier interferograms (see the 3-ns frame) show an expanding plasma with a density distribution that presents a maximum on axis. However, as time evolves and the pump laser inten- sity increases, the plasma density profile acquires a concave shape with a density minimum on axis, which is already clearly developed at $6 \mathrm{~ns}$ after the initiation of the current pulse, and that becomes more pronounced in the subsequent several nanoseconds. The asymmetry observed in some of the interferograms is due to a slight misalignment of the target. At times after termination of the Nd:YAG laser pulse, the decreasing degree of ionization of the plasma causes significant absorption of the probe beam by photoionization. Measurements were conducted to verify that the observed density profile is not a consequence of the intensity profile of the pump laser. It should be noticed that due to the large scale length of the plasma and large density gradients, an ultraviolet laser probe (e.g., fourth harmonic of Nd:YAG at $266 \mathrm{~nm}$ ) would be very strongly refracted.

To understand this unusual electron density profile, we conducted simulations with the hydrodynamic code LASNEX [15]. The severe distortion of the plasma motion inferred from our SXRL interferograms can be well described only by 2D simulations. Both single-dimension (1D) and 2D modeling rule out the possibility that the ponderomotive force might be the primary cause of the observed plasma behavior, as it is known to take place for larger fluxes [1] or with long wavelength laser irradiation [16]. The computed evolution of the plasma density profile is shown in Fig. 4. The resulting 


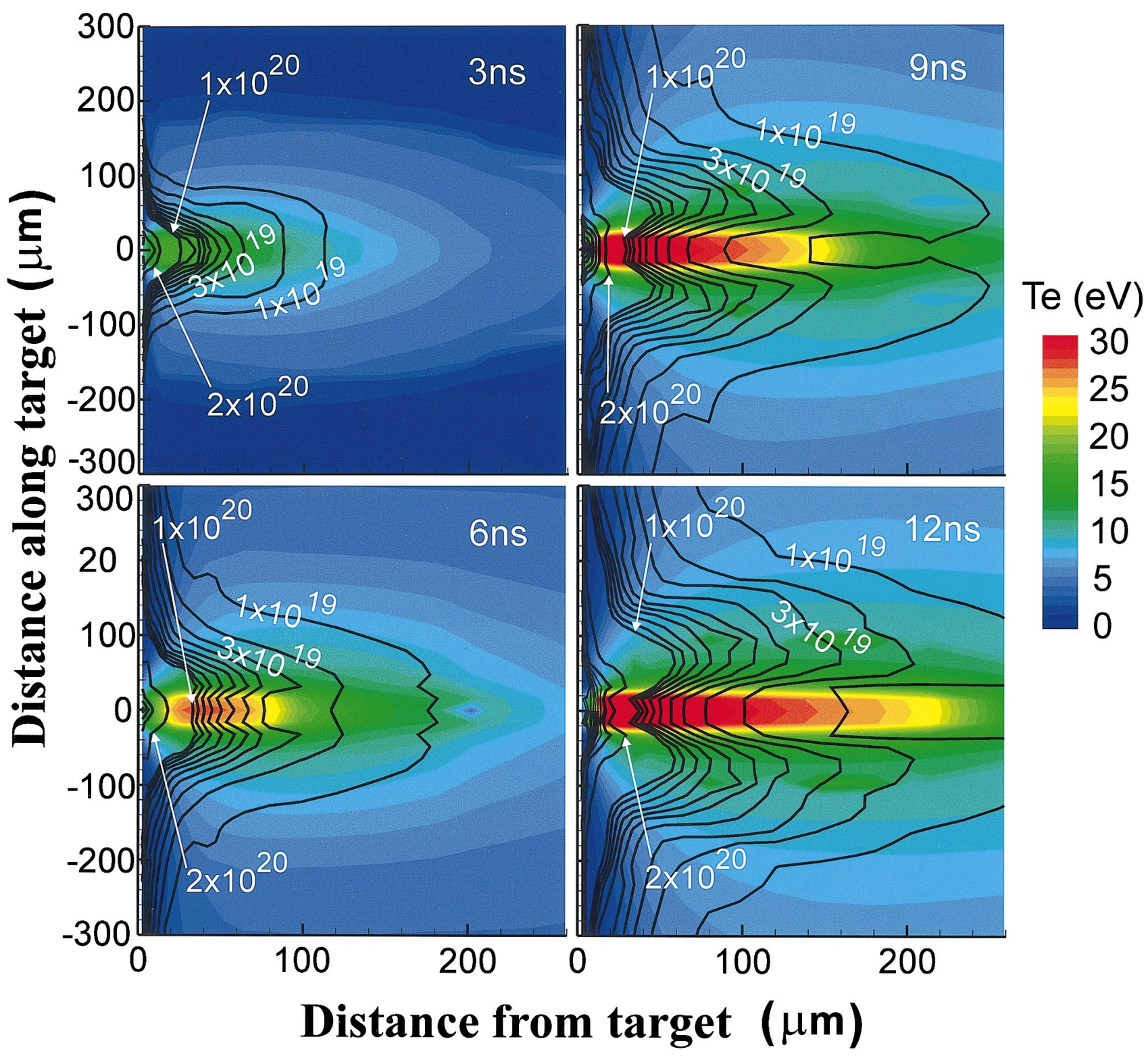

FIG. 4. (Color) Simulated electron density (line contours) and temperature (filled contour) profiles of the line-focus plasma of Fig. 3 computed using LASNEX. The heating laser is incident from the right. The calculations were done for a $10^{11} \mathrm{~W} / \mathrm{cm}^{2}$, $13-\mathrm{ns}-\mathrm{FWHM}$ laser pulse.

physical picture consists of a relatively high temperature $(\sim 36 \mathrm{eV})$ central region surrounded by a lower temperature $(\sim 10 \mathrm{eV})$ plasma on either side. The on-axis density distribution and velocity fit well with the results of both $1 \mathrm{D}$ cylindrical expansion and 2D simulations of a laser-irradiated plasma. Instead, the sidelobes are entirely 2D formations created outside the laser-irradiated target region by the buildup of new cold material resulting mainly from extreme ultraviolet (XUV) plasma radiation induced evaporation. In addition, 2D simulations show that radiation cooling contributes in substantially lowering the temperature of the sidelobes. The lobes have slower axial expansion $\left(<1 \times 10^{6} \mathrm{~cm} \mathrm{~s}^{-1}\right)$ as compared to the hotter central plasma region (5-6 $\left.\times 10^{6} \mathrm{~cm} \mathrm{~s}^{-1}\right)$. After several nanoseconds the pressure balances in the lateral direction within a distance of $\sim 100 \mu \mathrm{m}$ from the axis, as dictated by the sound's speed $\left(\sim 10^{6} \mathrm{~cm} \mathrm{~s}^{-1}\right)$ and plasma lifetime $\left(\sim 10^{-8} \mathrm{~s}\right)$, hence forming a density depression on axis and lobes in the colder areas. To illustrate that the sidelobes are mainly caused by outside spot ablation, we conducted simulations in which we constrained the target ablation outside the area illuminated by the laser, while maintaining otherwise identical physics. The result in Fig. 5 (right) shows that in this case the expansion is almost classical and presents significantly smaller density inhomogeneities. Furthermore, an additional simulation shows that if all radiation effects are excluded the expansion becomes classical and the sidelobes disappear, in which case the temperature in the conical expansion does not drop as dramatically along the surface.

Our modeling shows that this 2D density behavior is essentially a universal effect. It includes the case of larger focal spots, but in this case it is not as pronounced. Some indica- 


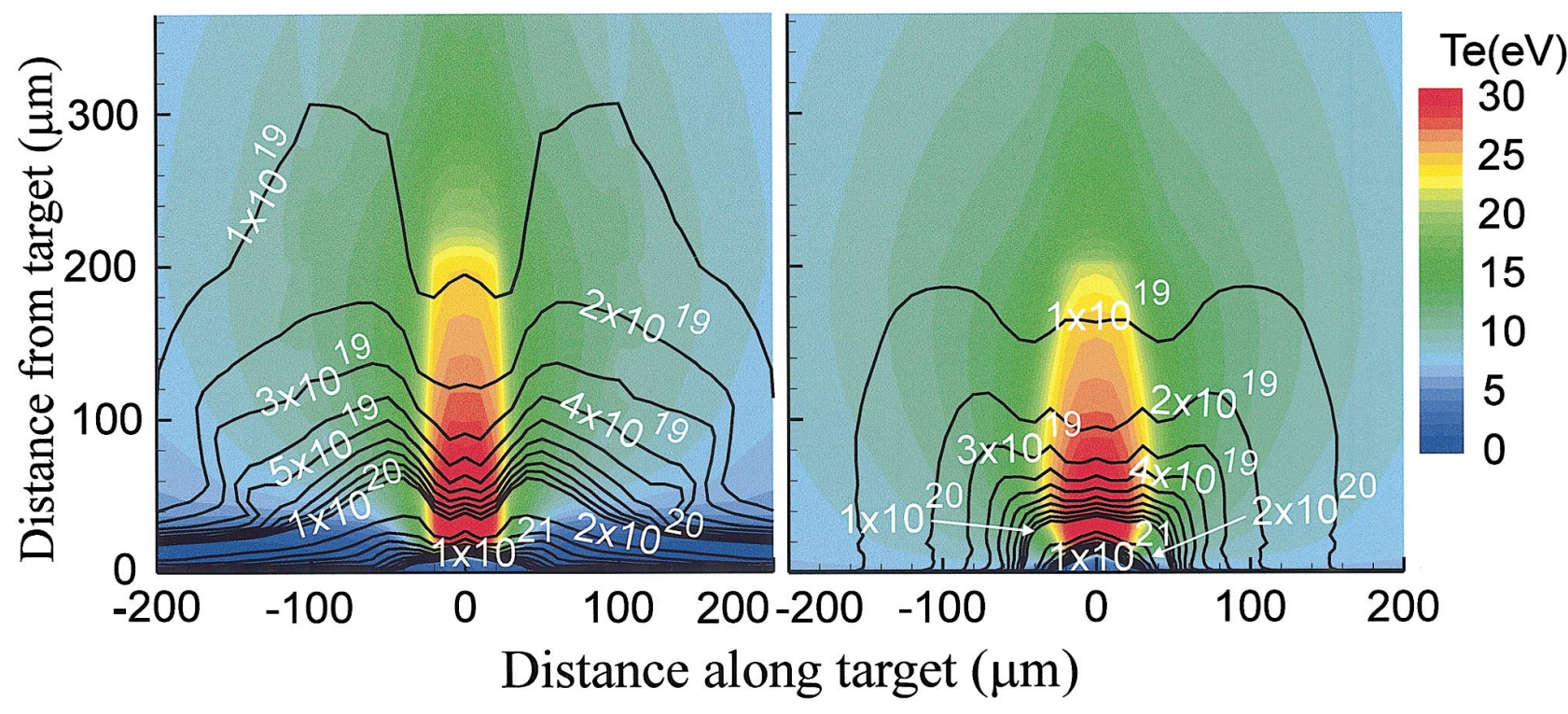

FIG. 5. (Color) Simulated density (line contours) and electron temperature (filled contour) profiles for the 12-ns-line-focus plasma (left), and with the motion of the ablated material from the target outside the spot constrained (right). Otherwise the conditions for the two simulations are identical. The heating laser is incident from the top. Both figures are calculated at the peak of the $10^{11} \mathrm{~W} / \mathrm{cm}^{2}, 13-\mathrm{ns}-\mathrm{FWHM}$ laser pulse.

tions to this effect can be found in the experiment of Bol'shov et al. [6]. Due to the fact that dense plasmas are very efficient sources of XUV radiation even at low plasma temperatures, this kind of plasma behavior should be seen in many experiments. However, because it takes a relatively long time $\sim 4-10 \mathrm{~ns}$ for the off-spot material to evaporate and the colder plasma to expand, this effect was not previously clearly identified in shorter pulse experiments. The longer pulse duration in the present experimental situation allows the establishment of the pressure balance, such that the hotter central plasma corresponds to a lower density.

A similar but even more pronounced density depression was observed in plasmas generated at increased irradiation intensity by focusing the Nd:YAG laser onto a spot $<30 \mu \mathrm{m}$ in diameter, using a $15-\mathrm{cm}$-focal-length aspheric lens. In this case the irradiation intensity was $7 \times 10^{12} \mathrm{~W} \mathrm{~cm}^{-2}$ and the plasma was imaged with $51 \mathrm{x}$ magnification. A series of interferograms were obtained for plasmas generated by either firing the laser onto a new target spot or after firing several laser shots in the same target location. In all cases, we observed the formation of similar density lobes and depression. However, the plasmas produced after multiple laser shots in the same target location are observed to develop larger density sidelobes at larger distances from the target. This results from the fact that these plasmas emanate from the crater created by the previous shots, which constrains its lateral expansion and guides the plasmas motion into the direction normal to the target. In this case the amount of plasma generated is substantially increased by the larger ablation caused by the higher temperature, the contact of plasma with the crater's wall and the increased XUV emission efficiency at higher densities. The modeling shows that despite almost two orders of magnitude larger laser fluxes than in the previous case, the plasma transport coefficients continue to be classical and the ponderomotive force is still negligibly small to have any effect on the plasma profile. Figure 6 shows an interferogram corresponding to a second shot in the same target location obtained near the time of peak laser intensity together with the corresponding electron density distributions resulting from Abel inversion. The formation of pronounced density peaks off-axis is again seen. In the region mapped by the interferograms the electron density is observed to increase as a function of time, reaching at the time of maximum laser intensity a value of $9 \times 10^{20} \mathrm{~cm}^{-3}$ (which amounts to about $90 \%$ of the critical density for the $\lambda$ $=1.06-\mu \mathrm{m}$ pump laser) at $27 \mu \mathrm{m}$ from the target. As in the case of the line-focus plasma, the experimental study of a seemingly typical laser-created plasma discloses a situation that cannot be well described by 1D hydrodynamic simula-
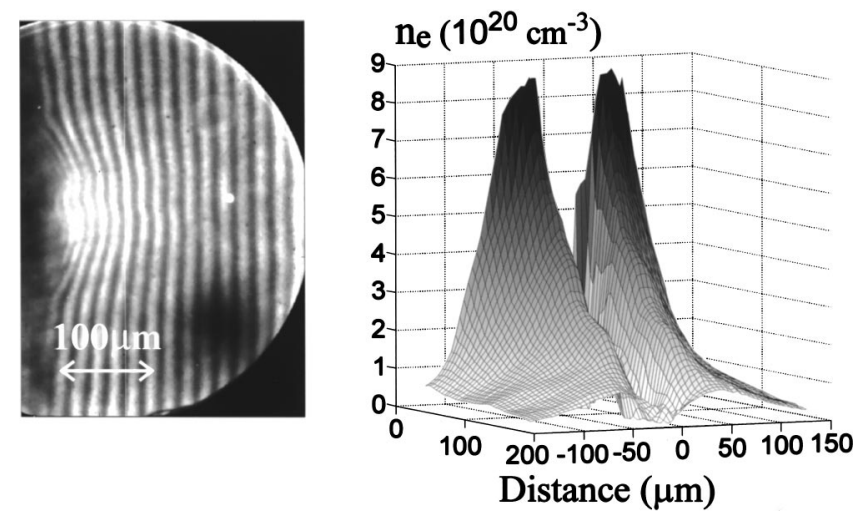

FIG. 6. Measured soft-x-ray interferogram and corresponding electron density profile for a spot focus plasma. The plasma was generated focusing a 13-ns-FWHM pulse of $0.63 \mathrm{~J}$ energy into a $30-\mu \mathrm{m}$-diameter spot. The interferogram was acquired at a time near the peak intensity of the heating laser pulse. 
tions, though again 1D codes are found to reproduce qualitatively well the central region of the plasma expansion.

In conclusion, we have observed 2D hydrodynamic effects of large magnitude in laser-created plasmas generated at relatively low irradiation intensities leading to the formation of concave electron density profiles. Simulations show that the buildup of cold mass outside the focal spot, combined with pressure balance results in the formation of the observed density depression. The results constitute the demonstration of the use of table-top SXRL interferometry in the study of complex high density plasma phenomena. The SXRL interferometry with compact table-top lasers is positioned to become an important high-resolution diagnostic, not only to measure density profiles in high density plasmas but also for the development and validation of hydrodynamic codes and plasma theory.

The authors would like to thank G. B. Zimmerman, D. C. Eder (LLNL), N. G. Kovalsky, A. E. Stepanov (TRINITI), A. Ya. Faenov (VNIIFTRI), and Yu. A. Zakharenkov for valuable discussions. This work was supported by U.S. Department of Energy Grant No. DE-FG03-98DP00208 and by the National Science Foundation. Part of this work was performed under the auspices of the U.S. Department of Energy by the University of California, Lawrence Livermore $\mathrm{Na}$ tional Laboratory under Contract No. W-7405-Eng-48. We also gratefully acknowledge the support of the W. M. Keck Foundation.
[1] D. T. Attwood et al., Phys. Rev. Lett. 40, 184 (1978).

[2] S. Wilks et al., Phys. Rev. Lett. 73, 2994 (1994).

[3] K. Takahashi et al., Phys. Rev. Lett. 84, 2405 (2000).

[4] C. E. Max, C. F. McKee, and W. C. Mead, Phys. Rev. Lett. 45, 28 (1980).

[5] G. S. Sarkisov, Phys. Rev. E 59, 7042 (1999).

[6] Bol'shov et al., Sov. Phys. JETP 65, 1160 (1987).

[7] Yu. A. Zakharenkov et al., Sov. Phys. JETP 70, 547 (1976); Yu. A. Zakharenkov (private communication).

[8] L. B. Da Silva et al., Phys. Rev. Lett. 74, 3991 (1995).
[9] A. S. Wan et al., Phys. Rev. E 55, 6293 (1997).

[10] J. Filevich et al., Opt. Lett. 25, 356 (2000).

[11] Y. Liu et al., Phys. Rev. A 63, 033802 (2001).

[12] B. R. Benware et al., Phys. Rev. Lett. 81, 5804 (1998).

[13] C. D. Macchietto, B. R. Benware, and J. J. Rocca, Opt. Lett. 24, 1115 (1999).

[14] Yu. A. Uspenskii et al., Opt. Lett. 23, 771 (1998).

[15] G. D. Zimmerman and W. L. Kruer, Comments Plasma Phys. Controlled Fusion 2, 51 (1975).

[16] V. Yu. Baranov et al., Laser Part. Beams 14, 347 (1996). 